\title{
On Modern Technical Approaches of Three- Dimensional High-Dose Lattice Radiotherapy (LRT)
}

\author{
Xiaodong $\mathrm{Wu}^{1}$, Mansoor M. Ahmed ${ }^{2}$, Jean Wright ${ }^{3}$, Seema Gupta ${ }^{3}$, Alan Pollack $^{3}$ \\ 1. CyberKnife Center of Miami; University of Miami Miller School of Medicine, Miami, FL 2. Department \\ of Radiation Oncology, Miami University 3. Department of Radiation Oncology, University of Miami, \\ Miller School of Medicine, Miami, Florida
}

$\square$ Corresponding author: Xiaodong Wu, xwu@med.miami.edu

Disclosures can be found in Additional Information at the end of the article

\section{Abstract}

Purpose: Two-dimensional (2D) high-dose GRID radiotherapy has shown effective tumor control in the clinical setting, and radiobiological data suggest potent bystander/abscopal effects with this technique. We present a new technical concept using modern radiation therapy instrumentation to advance traditional GRID treatment to modern three-dimensional (3D) high-dose LATTICE radiotherapy (LRT).

Materials and Methods: An array of focused high-dose volumes, in essence a lattice of doses in 3D, can be generated through modern techniques resulting in highly heterogeneous dose distributions within the tumor volume, leaving adjacent and peripheral normal tissue minimally exposed. Two technical approaches were investigated for this novel concept: noncoplanar focused beams and MLC-based or aperture-modulated arc.

Results: High magnitude dose oscillation is essential in traditional GRID therapy. The 3D "peak-to-valley" dose fall-off (100\% to 20-30\%) characteristic was attainable in test LRT cases, with a greater ability to place dose peaks within the radiation target volume and minimal dose to surrounding normal tissue.

Conclusion: Modern radiotherapy methods are readily available to deliver 3D high-dose LATTICE radiotherapy with superior dosimetry compared to the 2D GRID technique. This is an easily accessible therapeutic modality that could potentially result in comparable or superior treatment outcomes than traditional 2D GRID therapy when implemented in the clinical setting.

Published 03/05/2010

C) Copyright 2010

Wu et al. This is an open access article distributed under the terms of the Creative Commons Attribution License CC-BY 3.0., which permits unrestricted use, distribution, and reproduction in any medium, provided the original author and source are credited.
Categories: Radiation Oncology

Keywords: non-coplanar focused beams, cyberknife, lattice radiotherapy (lrt), bystander/abscopal effect, grid therapy

\section{Introduction}

High-dose GRID radiotherapy, sometimes termed spatially-fractionated GRID radiotherapy (SFGRT), remains a less frequently used treatment modality since its inception in the early 1930 's [1]. In its early applications, two-dimensional GRID fields were used, typically with orthovoltage beams allowing spatially alternated dose distributions [2-3]. The grids were usually composed of a shield with an array of openings of circular or square shapes ranging in size from 
0.5 to $1.5 \mathrm{~cm}$. The application was reserved mainly for the treatment of advanced bulky tumors. It was argued that such a treatment technique permitted higher dose delivery with acceptable skin toxicity due to the fact that highly exposed skin regions are surrounded by undamaged skin resulting in improved repair.

Although relatively small numbers of patients have received GRID treatment either with orthovoltage or more recently with MV X-rays, significant and dramatic tumor regressions have been observed and reported [4-7], even though GRID therapy, in contrast to conventional approaches, does not attempt to treat the total tumor volume with a relatively uniform dose.

Recent research in radiobiology has provided new biological insights. These include the bystander effect within the GRID irradiated tumor volume that occurs in the tumor cells that fall directly under shielded regions (low-dose regions) of the GRID. Bystander factors, such as TNF-a [8-9], TRAIL [9], and Ceramide [10], are induced in cells that are under the open field of the high-dose GRID areas and are hypothesized to be responsible for initiating the cell death cascade, both in the epithelial and endothelial compartments of the tumor micro-environment. In addition to the bystander effect within the GRID irradiated tumor, Peter, et al. [11] reported that there is a robust abscopal effect in distant tumors or metastatic lesions that are not irradiated or treated. These data strongly suggest that GRID therapy would induce a rapid and higher rate of tumor cell apoptosis in bulky and hypoxic tumors than conventional radiotherapy. It has been shown previously that a high dose given before the conventional radiation dose of $2 \mathrm{~Gy}$ results in increased cell killing in vitro [9] and increased tumor growth delay and regression in vivo (unpublished data). Therefore, we hypothesize that induction highdose GRID radiotherapy would enhance the therapeutic outcome of subsequent conventional or hypofractionated, dosimetrically-uniform radiation therapy. This new approach is a paradigm shift with the potential to significantly influence the outcome of patients with tumors that are more resistant to radiation therapy. We continue to investigate this hypothesis in the laboratory and clinical setting.

However, to move forward with clinical implementation of induction spatially fractionated radiation followed by standard radiation doses without anticipating increased treatment related toxicity, better techniques for the delivery of spatially fractionated radiation must be developed. Although effective with acceptable toxicity, 2D GRID treatments still expose a considerable volume of normal tissue to high radiation dose. In addition, the 2D GRID approach often delivers the highest radiation dose to superficial tissue that is not within the radiation clinical target volume. The 2D GRID can be reconfigured into a 3D dose lattice, whereby high doses are concentrated at each lattice vertex within the radiation target volume with drastically lower dose between vertices (peak-to-valley effect,) and leaving anything outside of tumor volume minimally exposed (Figure 1). The term "LATTICE" is introduced here only for figurative purposes; no rigorous requirement of symmetry is implied. Two technical approaches of achieving such high-dose lattice distribution are investigated in this study. 


\section{Cureus}

2D grid radiation field

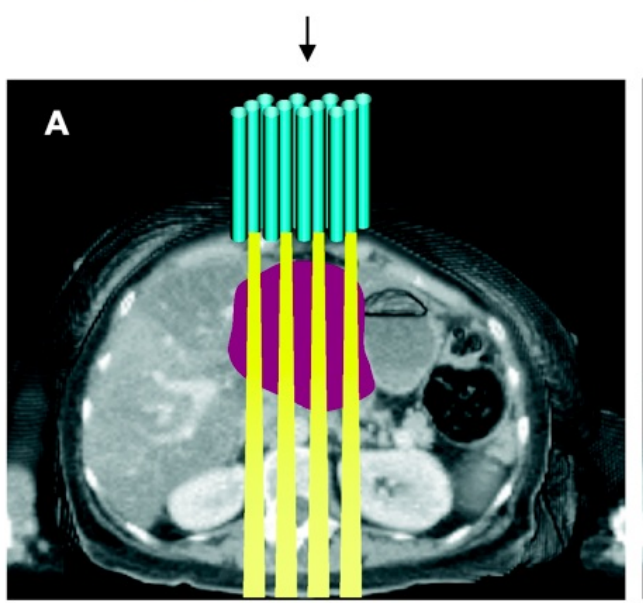

Focused beams to form dose lattice

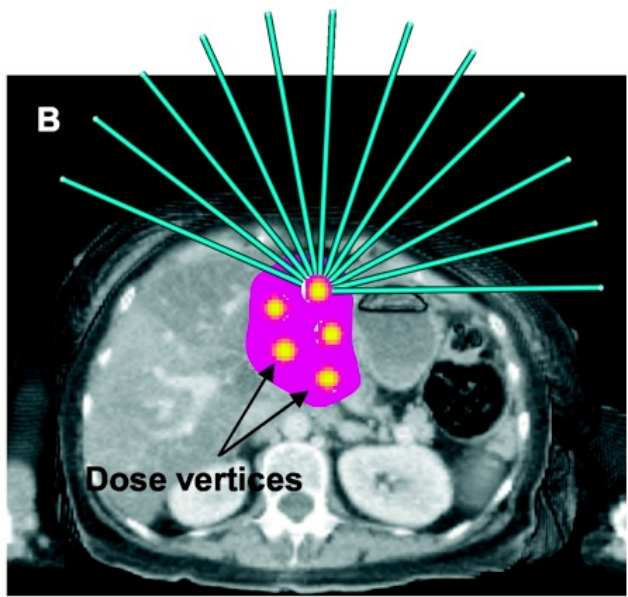

FIGURE 1: Conceptual comparison between the traditional 2D GRID and the 3D dose lattice

\section{Materials And Methods}

With modern radiation therapy instrumentation, it is readily possible to obtain highly localized 3D high-dose array or lattice within the tumor volume, in which highly concentrated, spherelike dose distributions are located in lattice vertices with a rapid dose fall-off between vertices resulting in a periodic peak-to-valley dose distribution in 3D. The proposed techniques for delivering high-gradient lattice dose distribution include IMRT or rotation delivery with dynamic-MLC linear accelerator, multiple focused non-coplanar beams with a roboticcontrolled linear accelerator, and proton/heavy ion beams with spot scanning nozzles. Currently available real-time target tracking technologies, such as the Synchrony (Accuray Inc.) and the Calypso 4D (Calypso Medical Inc.) or respiratory gating/controlling systems, can further insure the integrity of peak-to-valley effect of the dose lattice.

Two examples with focused photon beams are given to demonstrate the principle and the technical feasibility of the concept.

\section{Single or Multiple Arc with Dynamic MLC gantry-linear accelerator}

The 3D dose lattice can be generated with MLC-based IMRT or aperture-modulated arc technique [13]. Depending on the mechanical flexibility of the MLC used, multiple conformal arcs or a single arc, if inter-digitation is available, can be used. In this study, for comparison purposes, we applied a conventional static 2D GRID (Figure 2A) and a 3D LATTICE using the RapidArc technique (Varian Medical System, Inc.) (Figure 2B) to a large lung tumor of $390 \mathrm{cc}$. The 2D GRID had the commonly used hexagon configuration with $1 \mathrm{~cm}$ opening (diameter) and $1.8 \mathrm{~cm}$ separation (from center to center). In the LATTICE plan, 20 dose vertices were produced with separation of about $2 \mathrm{~cm}$, using 6 arcs with $18 \mathrm{MV}$ x-rays. The maximum dose was $18 \mathrm{~Gy}$ in both examples. Peak-valley dose distribution patterns were analyzed. DVHs of the GTV, critical structures and the body were generated for comparison. 


\section{Cureus}

2D Grid configuration
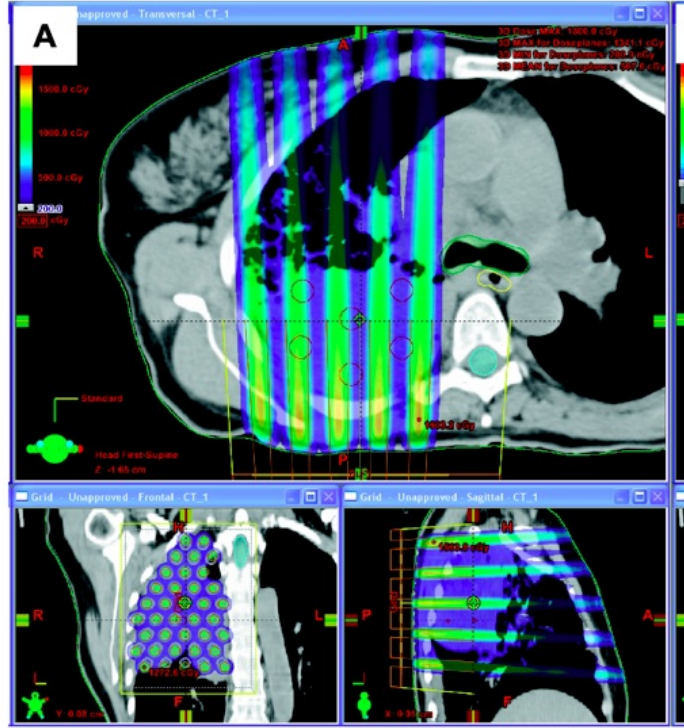

Lattice configuration

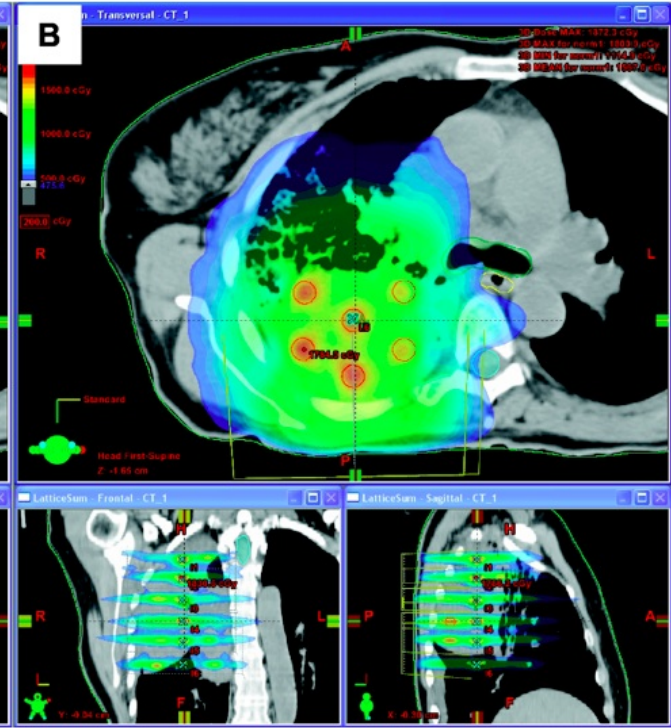

FIGURE 2: Isodose comparison between a 2D grid and an LRT with RapidArc, lung example

2. Non-coplanar focused beams

Many radiotherapy delivery systems are now capable of delivering multiple focused beams to form highly converged dose distribution. Such techniques are commonly used for stereotactic radiosurgery (SRS) or stereotactic body radiotherapy (SBRT). The second example shown herein is a 3D dose lattice in a $185 \mathrm{cc}$ pancreatic mass, achieved through the CyberKnife (Accuray Inc.), an image-guided robotic radiosurgery system [12]. Eleven vertices (isocenters) are distributed throughout the tumor volume with about $2.5 \mathrm{~cm}$ of separation. Beams from 40-50 non-coplanar directions with a $7.5 \mathrm{~mm}$ collimator were used for each isocenter. The objective was again to create a highly heterogeneous, oscillating dose distribution of which peak-tovalley dose could fall from $100 \%$ to $20-30 \%$. Figure $3 A$ shows the configuration of the robotically controlled beams sequentially focused on each isocenter (vertex). DVH and peakvalley dose distribution were analyzed. Real-time tracking was used for delivering the dose plan. 


\section{Cureus}
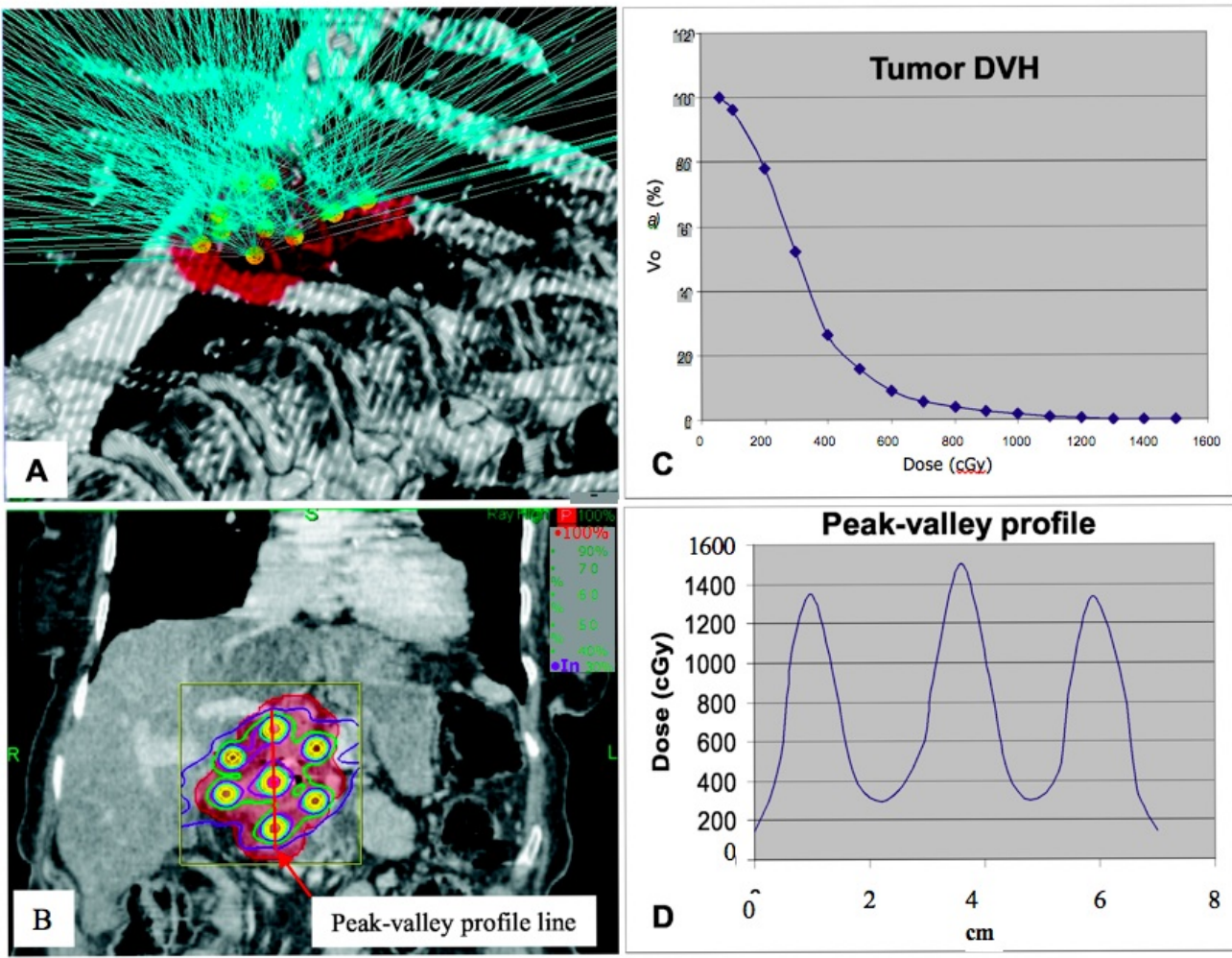

FIGURE 3: LATTICE RT with CyberKnife, pancreatic example

\section{Results}

In the first example, Figure 4 demonstrates a comparative dose-volume histogram (DVH) for the GRID plan and the LATTICE plan for treatment of a large lung mass, in which $18 \mathrm{~Gy}$ is prescribed to Dmax in both. The LATTICE plan is generated using RapidArc IMRT technology. With respect to coverage of the tumor volume (GTV), the overall shape of the DVHs are similar, with LATTICE (triangles) resulting in higher maximum dose to the GTV, and a greater volume of the GTV receiving high-dose radiation compared to GRID. The comparative dose to normal tissues with GRID versus LATTICE would be expected to vary significantly with tumor location in relation to normal structures. In this case, the structure that is best spared with LATTICE compared to GRID is normal lung. While GRID results in slightly less lung volume receiving the lowest radiation dose (<1 Gy), LATTICE exposes a lower volume of lung tissue to doses above 1 Gy than GRID. In this case, doses to the spinal cord and esophagus are slightly lower using GRID than LATTICE, but in tumors that are closer to midline or have gross mediastinal lymph node involvement, LATTICE is obviously superior to GRID for both spinal cord and esophagus. 


\section{Cureus}

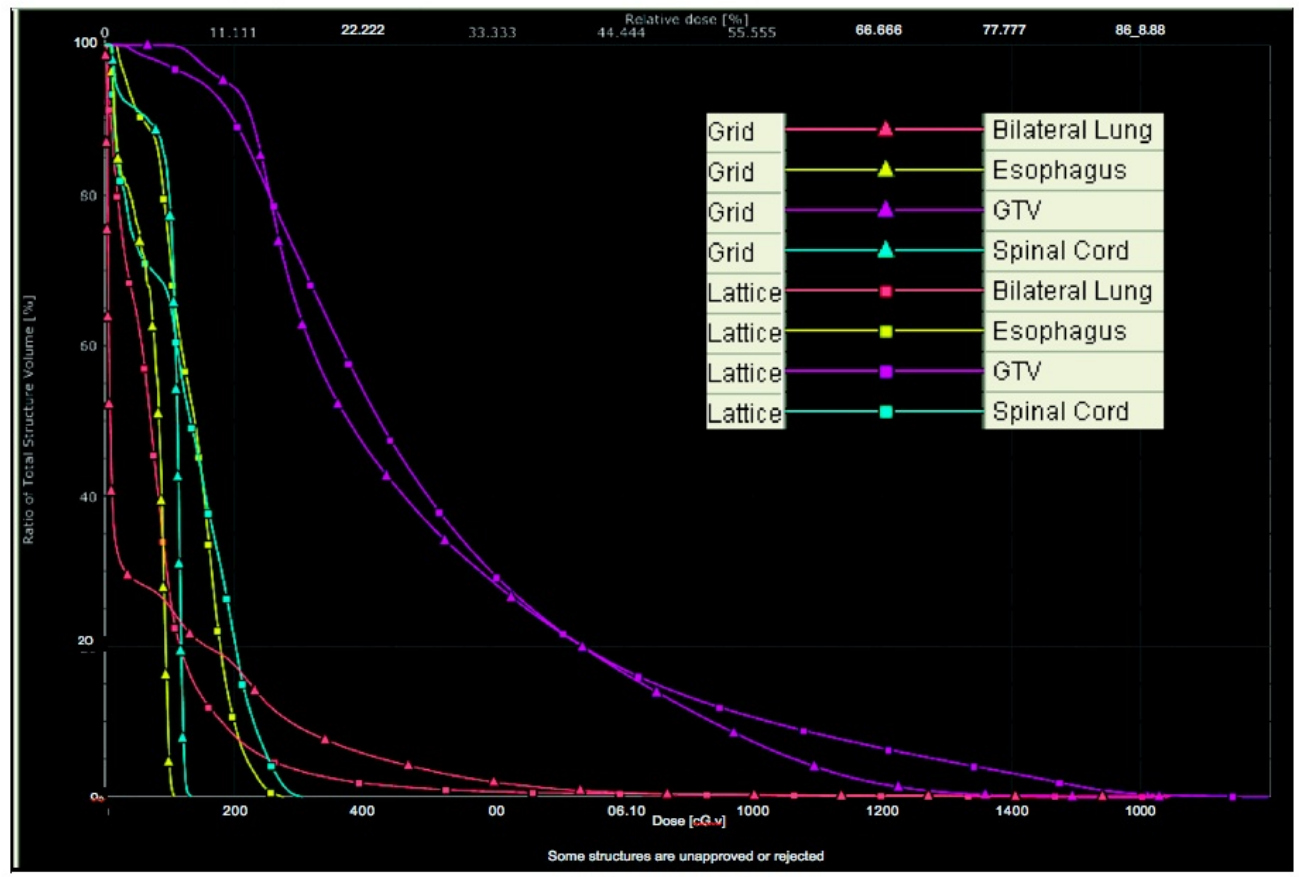

FIGURE 4: DVH Comparison Between GRID \& LATTICE--Iung example

It is important to note that with the LATTICE technique all high-dose islands are within the tumor target, and that dosefall-off outside these islands is rapid, resulting in low-dose valleys within the tumor as well as low radiation dose outside of the target volume. In comparison, 2D GRID results in high-dose pipes; however, the highest dose regions of these pipes are very superficial and occur outside the tumor target. Figure 5 demonstrates peak-valley dose profiles for GRID versus LATTICE. 


\section{Cureus}

A. 2D Grid configuration

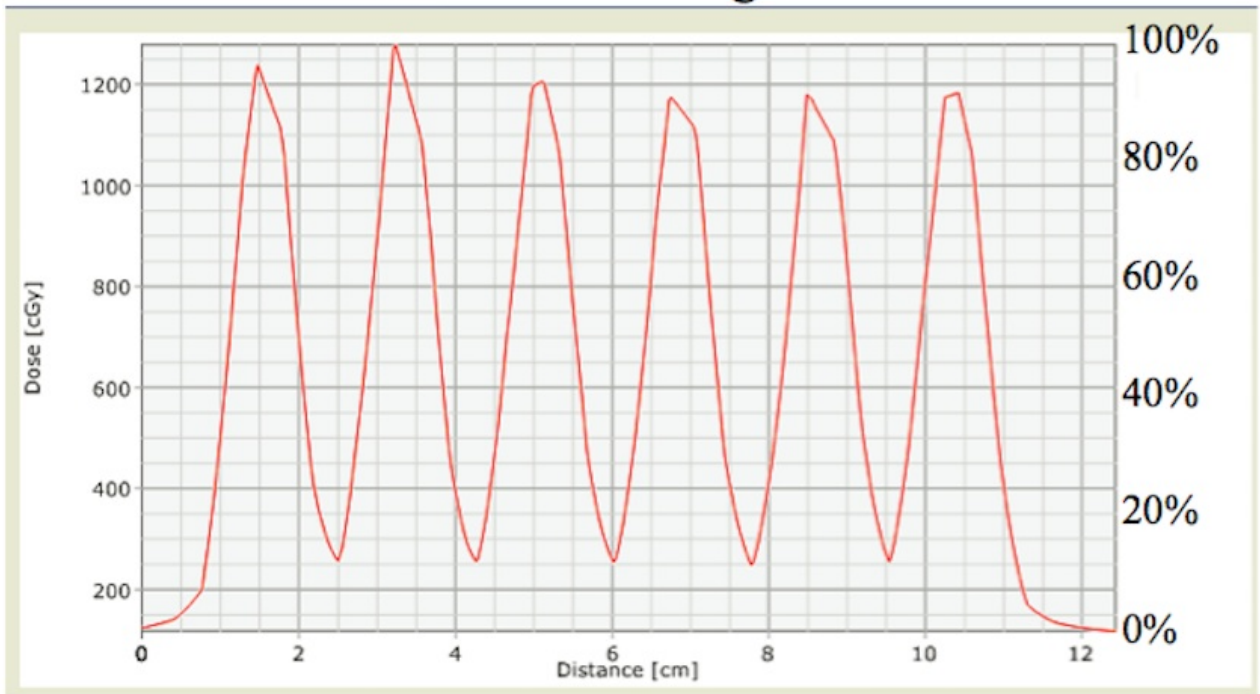

\section{B. Lattice configuration}

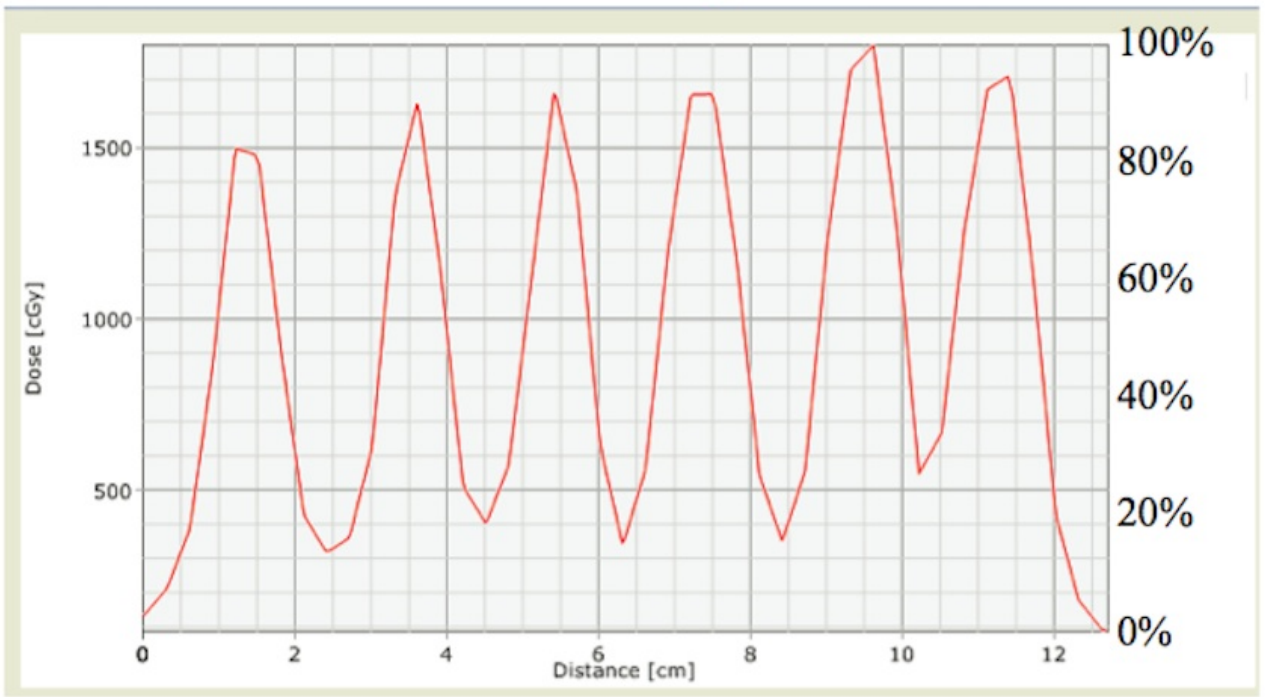

FIGURE 5: Peak-Valley Dose Profiles (GTV mid-plane)--lung example

Both GRID and LATTICE techniques result in pronounced radiation dose peaks and valleys, as is the goal of spatially fractionated radiation. While the dose in the valleys, or low-dose regions, is similar with both techniques, again the maximum dose falls within the tumor volume using LATTICE, while with GRID, they often fall outside the tumor volume. For the pancreatic mass example, the treatment plan is generated with multiple focused non-coplanar beams with a robotic-controlled linear accelerator using the CyberKnife technology. The high doses are also confined within the tumor volume, and the dose fall-off between vertices is shown to be 20$30 \%$ of the peak dose (Figure 3B, 3D). Figure 3C shows that the target DVH has a similar characteristic as shown in the first example (GRID vs RapidArc).

In both the RapidArc and CyberKnife LATTICE examples, highly heterogeneous dose 


\section{Cureus}

distributions are observed and are expected to be sufficient to trigger the expected biological effect. Only a small amount of the surrounding normal tissue/critical organs received 2-3 Gy or less.

These two examples were developed on the clinically commissioned commercial planning systems with no software and hardware modification. To affirm deliverability and accuracy, a CyberKnife-based LATTICE dose plan was delivered to a 3D QA phantom (Figure 6A, 6B). Widerange Gafchromic films were employed for dosimetric verification (Figure 6C). Good agreement ( $3 \%$ and $<1 \mathrm{~mm}$ ) between the measurements and the plans were obtained, as shown in Figure $6 D$ (computation in dash vs. measurement in color bands).
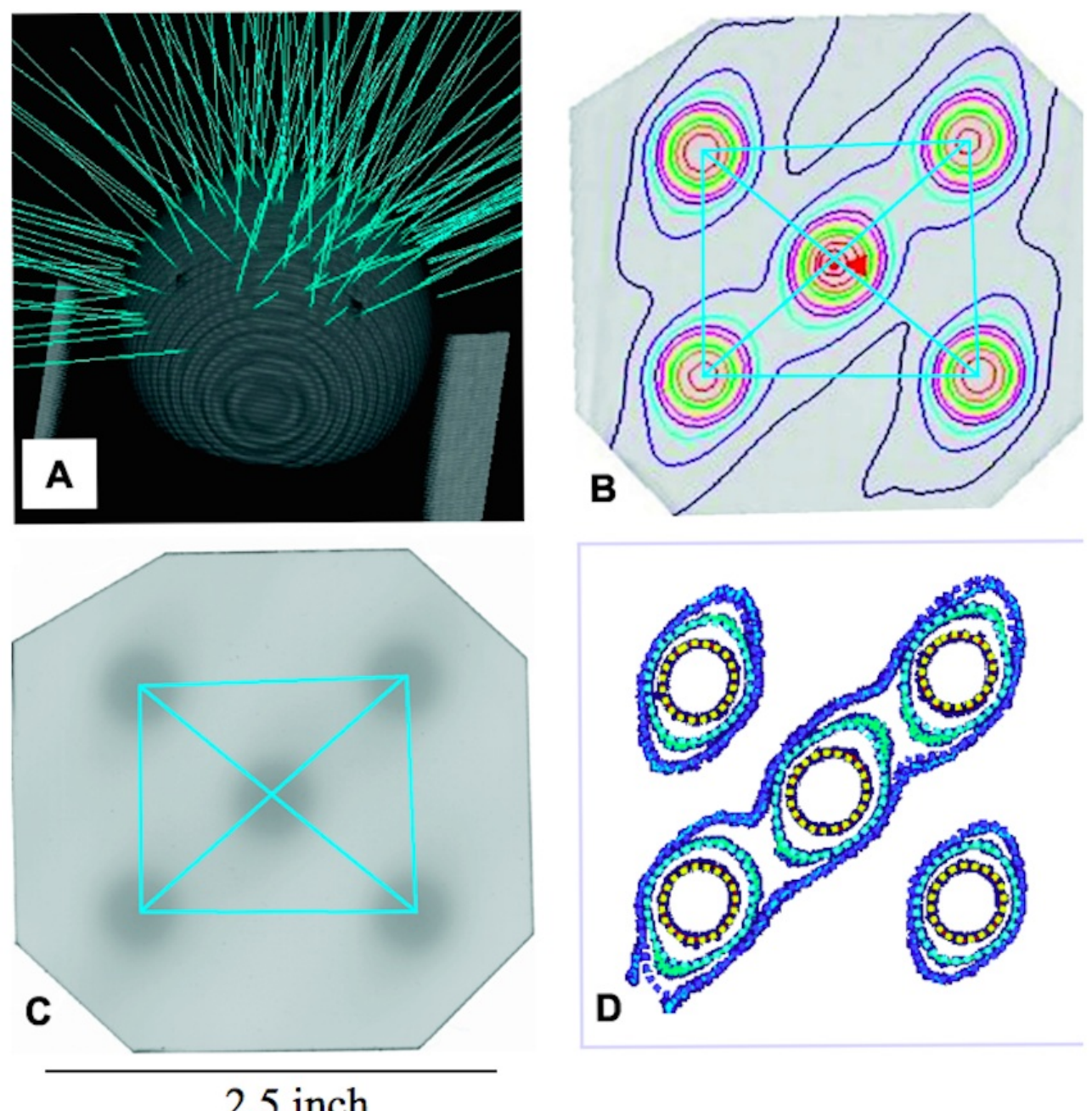

\section{5 inch}

FIGURE 6: CyberKnife LATTICE test on a phantom

\section{Discussion}

The peak-to-valley dose characteristic is thought to be essential to attain the GRID therapeutic effect. The transition from traditional 2D GRID treatment technique to the new 3D LATTICE technique is not only achievable but also advantageous in reducing the peripheral dose which mostly falls in normal tissue or critical organs. Further biological and dosimetric exploration is needed to determine the optimal configuration of a 3D dose lattice. To create a sufficient peakto-valley dose differentiation, minimal separation between dose vertices is required and is 


\section{Cureus}

dictated by the number of high dose vertices and the size of focused beams or the vertex high dose volume (12 Gy and higher). More vertices or larger high dose volume would require wider vertex separation. It should be noted that the vertex dose distribution need not to be spherical or near spherical. The basic principle of the LATTICE Radiotherapy (LRT) is to create within tumor volume multiple localized high-dose islands (12 Gy and higher) with a certain degree of separation to form low dose regions (3 cGy or lower). In an extreme case, one or two focused dose islands could be introduced in a small tumor.

Heavy charged particle beams, such as proton and carbon ion beams, have gained wider interest in the recent years [14]. A spot-scanning technique has been developed to confirm the prescribed dose to irregularly shaped 3D tumor volumes. Although not presently accessible to the authors, we postulate that a particle beam therapy system with a spot-scanning nozzle can be used to deliver 3D high-dose lattice radiotherapy. In this approach, a preferred set of the lattice parameters (number and locations/separation of the vertices) is first determined. The scanning nozzle is then programmed to deliver the Bragg Peak (or a minimally spread-out Bragg Peak) to each high-dose vertex (Figure 7).

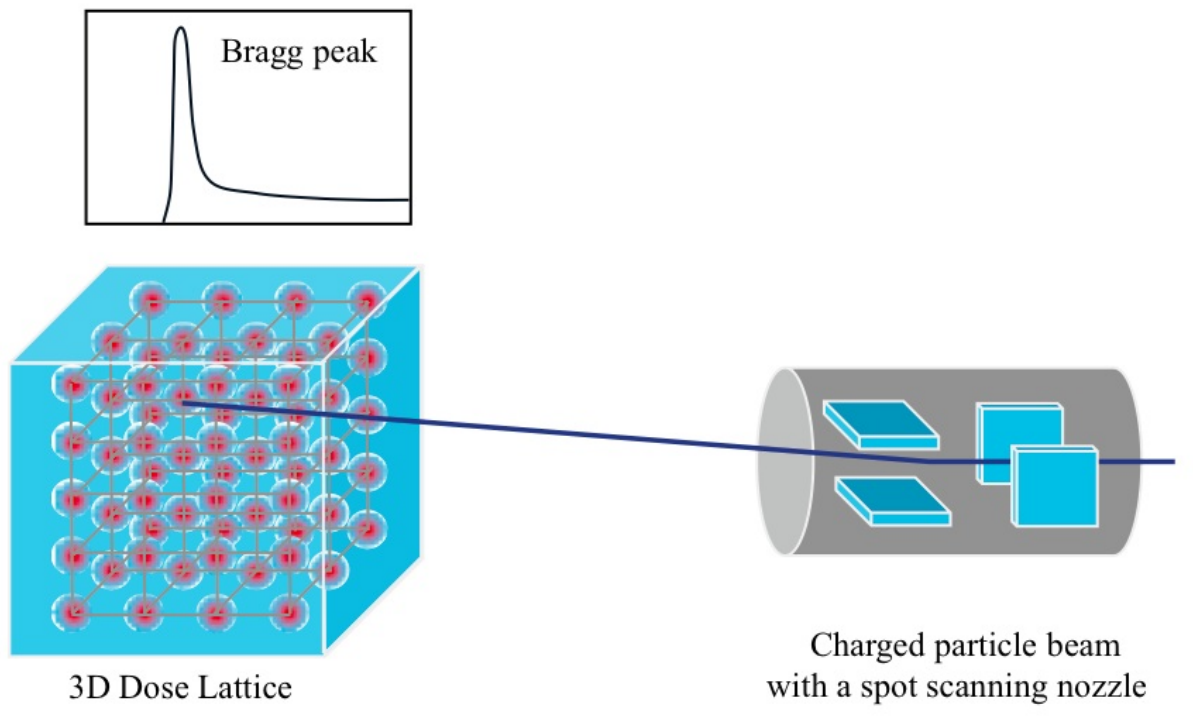

\section{FIGURE 7: A conceptual schematics of 3D LATTICE formation by charged particle beam}

It is worth noting that the spatially fractionated radiation in a rather extreme form has also been investigated with microbeam radiation therapy (MRT), which uses bundles of highintensity synchrotron $\mathrm{x}$-ray micro beams in the 50-150 keV energy range, with a typical width of 25 ,um and a 200 ,um distance between beams [15-17]. A similar rationale of normal tissue sparing and bystander effects is hypothesized. MRT is, however, generally not attainable in conventional radiation therapy facilities.

\section{Conclusions}

To date, 2D GRID therapies have been offered to patients with advanced and bulky tumors who had no other possible treatment options. With encouraging clinical outcomes, it is hoped that the 3D LATTICE Radiotherapy technique described herein could offer the same benefit to more 
patients with less toxicity. Beyond this, if the new insights into the radiobiology of spatially fractionated radiation are shown to have clinical relevance, a new paradigm of using high-dose (12 Gy and higher) 3D LRT as induction therapy, followed by conventional radiation therapy or chemotherapy, can be anticipated.

\section{Additional Information \\ Disclosures}

Human subjects: All authors have confirmed that this study did not involve human participants or tissue. Animal subjects: All authors have confirmed that this study did not involve animal subjects or tissue. Conflicts of interest: In compliance with the ICMJE uniform disclosure form, all authors declare the following: Payment/services info: This work was supported by grant from NCI (R01CA86937). Financial relationships: All authors have declared that they have no financial relationships at present or within the previous three years with any organizations that might have an interest in the submitted work. Other relationships: All authors have declared that there are no other relationships or activities that could appear to have influenced the submitted work.

\section{References}

1. Liberson F: The value of a multi-perforated screen in deep X-ray therapy. Radiology. 1933, 20:186-95.

2. Marks H: Clinical experience with irradiation through a GRID. Radiology . 1952, 58:338-42.

3. Freid JR, Lipman A, Jacobson LE: Roentgen therapy through a grid for advanced carcinoma. Amer J Roentgen . 1953, 70:460.

4. Mohiuddin M, Stevens JH, Reiff JE, et al.: Spatially fractionated (GRID) radiation for palliative treatment of advanced cancer. Radia Oncol Invest . 1996, 4:41-47.

5. Mohiuddin M, Fujita M, Regine WF, et al: High-dose spatially fractionated radiation (GRID): A new paradigm in the management of advanced cancer.. Int J Radiat Oncol Biol Phys . 1999, 45:721-27.

6. Zwicker RD, Meigooni A, Mohiuddin M: Therapeutic advantage of GRID irradiation for large single fractions. Int J Radiat Oncol Biol Phys . 2004, 58:1309-15.

7. Neuner GA, Vander Walde N, Ha JK, et al.: High-dose Spatially-fractionated GRID radiation therapy (SFGRT): A comparison of outcomes of treatment delivered through Cerrobend GRID versus MLC GRID. Int J Radiat Oncol Biol Phys . 2008, 72:5488.

8. Sathishkumar S, Dey S, Meigooni AS, et al: The impact of TNF-alpha induction on therapeutic efficacy following high dose spatially fractionated (GRID) radiation. Technology in Cancer Research Treatment . 2002, 1:141-47.

9. Shareef MM, Cui N, Burikhanov R, et al.: Role of TNF-a and TRAIL in high dose radiationinduced bystander signaling in lung adenocarcinoma. Cancer Research . 2007, 67:11811-20.

10. Sathishkumar S, Boyanovsky B, Karakashian AA, et al.: Elevated Sphingomyelinase Activity and Ceramide Concentration in Serum of Patients Undergoing High Dose Spatially Fractionated Radiation Treatment: Implications for Endothelial Apoptosis. Cancer Biol Ther. 2005, 4:979-86.

11. Peters ME, Shareef MM, Gupta S, et al.: Potential Utilization of Bystander / AbscopalMediated Signal Transduction Events in the Treatment of Solid Tumors. Current Signal Transduction Therapy. 2007, 2:129-43.

12. Brown WT, Wu X, Amendola B, Fowler JF, et al.: Treatment of early non-small cell lung cancer, Stage IA, by image-guided robotic stereotactic radioablation - CyberKnife. The Cancer Journal . 2007, 13:87-94.

13. Crooks SM1, Wu X, Takita C, et al.: Aperture Modulated Arc Therapy. Phys Med Biol. 2003, 48:1333-44.

14. Shulz-Ertner D, Tsujii H: Particle therapy using proton and heavier ion beams. J Clin Oncol . 2007, 25:953-64.

15. Slatkin DN, Spanne PO, Dilmanian FA, Sandborg M: Microbeam radiation therapy. Med. Phys. 1992, 19:1395-1400. 


\section{Cureus}

16. Slatkin DN, Spanne PO, Dilmanian FA, Gebbers JO, Laissue JA: Subacute neuropathological effects of microplanar beams of x-rays from a synchrotron wiggler. Proc. Natl Acad. Sci. 1995, 92:8783-87.

17. Brauer-Krisch E, Requardt H, R'egnard P, Corde S, Siegbahn E, LeDuc G, Brochard T, Blattmann H, Laissue J, Bravin A: New irradiation geometry for microbeam radiation therapy . Phys. Med. Biol. 2005, 50:3103-11. 